\title{
Kearns-Sayre Syndrome: A Rare Mitochondrial Disorder
}

\author{
QUAZI TARIKUL ISLAM, ${ }^{1}$ HOMAYRA TAHSEEN HOSSAIN, ${ }^{2}$ MD. ABUL KASHEM KHANDAKER, ${ }^{3}$ HAM NAZMUL \\ AHASAN, ${ }^{1}$ MAKSUDUL MAJUMDER,${ }^{4}$ ISHRAT BINTE REZA, ${ }^{4}$ TAJNUVA JABEEN,${ }^{5}$ KHADDOKER MORSHEDUL ALAM ${ }^{6}$
}

\begin{abstract}
:
Mitochondrial disease, once thought to be a rare clinical entity, is now recognized as an important cause of a wide range of neurologic, muscle, cardiac and endocrine disorders. Kearns Sayre syndrome is a rare mitochondrial disease, involving deletion of mitochondrial DNA. This syndrome ischaracterized by progressive external ophthalmoplegia (PEO), retinitis pigmentosa and an onset before the age of 20 years. First case was reported in 1958. We are reporting a case with chronic progressive external ophthalmoplegia, bilateral partial ptosis with onset at 10 years of age. He also had features of myopathy and neuropathy without any fatigable weakness. Our diagnosis is mostly based on clinical background and by exclusion of other common disorders, as definitive diagnostic investigation genetic testing due to unavailability so was not done.
\end{abstract}

Key Words: Mitochondrial disease, Kearns Sayre syndrome, progressive external ophthalmoplegia.

\section{Introduction:}

Kearns-Sayre syndrome (KSS) is a variety of mitochondrial disease characterized by progressive external ophthalmoplegia (PEO), pigmentary retinitis and an onset before the age of 20 years. Mitochondrial diseases are a clinically heterogeneous group of disorders that result from a dysfunction in the mitochondrial respiratory chain. ${ }^{1}$ Mitochondrial disorders are the most common form of inherited metabolic disorders. True prevalence is difficult to ascertain, as mitochondrial disease is almost certainly under reported. However, epidemiological evidence suggests a birth prevalence of 1 in 7634 live births and a lifetime risk of developing mitochondrial disease of approximately 1 in 5000.2,3

1. Professor, Medicine, Popular Medical College Hospital, Dhaka

2. Associate Professor, Medicine, Popular Medical College Hospital, Dhaka

3. Professor \& Head of Medicine, Popular Medical College Hospital, Dhaka

4. Registrar, Medicine, Popular Medical College Hospital, Dhaka

5. Asstt. Registrar, Medicine, Popular Medical College Hospital, Dhaka

6. Intern Doctor, Popular Medical College Hospital, Dhaka

Corresponding author: Prof. Quazi Tarikul Islam, Professor of Medicine, Popular Medical College Hospital, Dhaka. Email: prof.tarik@gmail.com.
Mitochondria are ubiquitous organelles and play crucial roles in vital functions, most importantly, the oxidative phosphorylation and energy metabolism. The tissues that require lots of energy have the most mitochondria, and so these highly energy-dependent tissues or organs are the ones most often affected in mitochondrial disease. Therefore, damage is most commonly presenting in the cells of the muscles, brain, heart, liver, gastrointestinal tract, ears and eyes. Due to a quirk in the process of fertilization, defects in the genes of the mtDNA are usually maternally transmitted. Patients with this disorder have a wide spectrum of symptoms due to varied genotype penetrance and disease severity. ${ }^{4}$ Patients may present with fibromyalgia, skeletal muscle weakness, ptosis, pain, fatigue, and exercise intolerance that progressively worsens over time, slowly progressive peripheral muscle weakness, multisystem organ failure or respiratory insufficiency requiring mechanical ventilation. 5,6

Due to the rarity ofthese disorders and variability in presentation, we are presenting our case report of a young male with features suggestive of mitochondrial myopathy, possibly Kearns-Sayre syndrome (KSS) to disseminate our clinical experience. High index of clinical suspicion is very essential for diagnosis of such cases due to limited facilities of definitive investigations like genetic testing.

\section{Case Report:}

A 20-year-old male was admitted into our department of 
JM Vol. 19, No. 1

Medicine for the evaluation of bilateral ptosis and external ophthalmoplegia for 10 years along with weakness of all four limbs for 5 years. The patient was in good health until the age of 10 . Since then, he insidiously developed drooping of both eyelids. This symptom was gradually progressive. There was no diurnal variation. Initially it was not associated with visual problem but with the time, patient noticed double vision on lateral gaze.

He also developed weakness of both upper and lower limbs for last 5 years. Weakness increased progressively, making it difficult to walk for last 3 years. For last 2 years, he had developed diminished sensation involving his hands and feet along with tingling and numbness. His bowel and bladder habit were normal. He did not complain of any hearing impairment. For last 2 years his ptosis, ophthalmoplegia and diplopia become worse.

He had a normal antenatal and perinatal history. His developmental milestone was age appropriate with normal intelligence. There is no history of consanguinity of

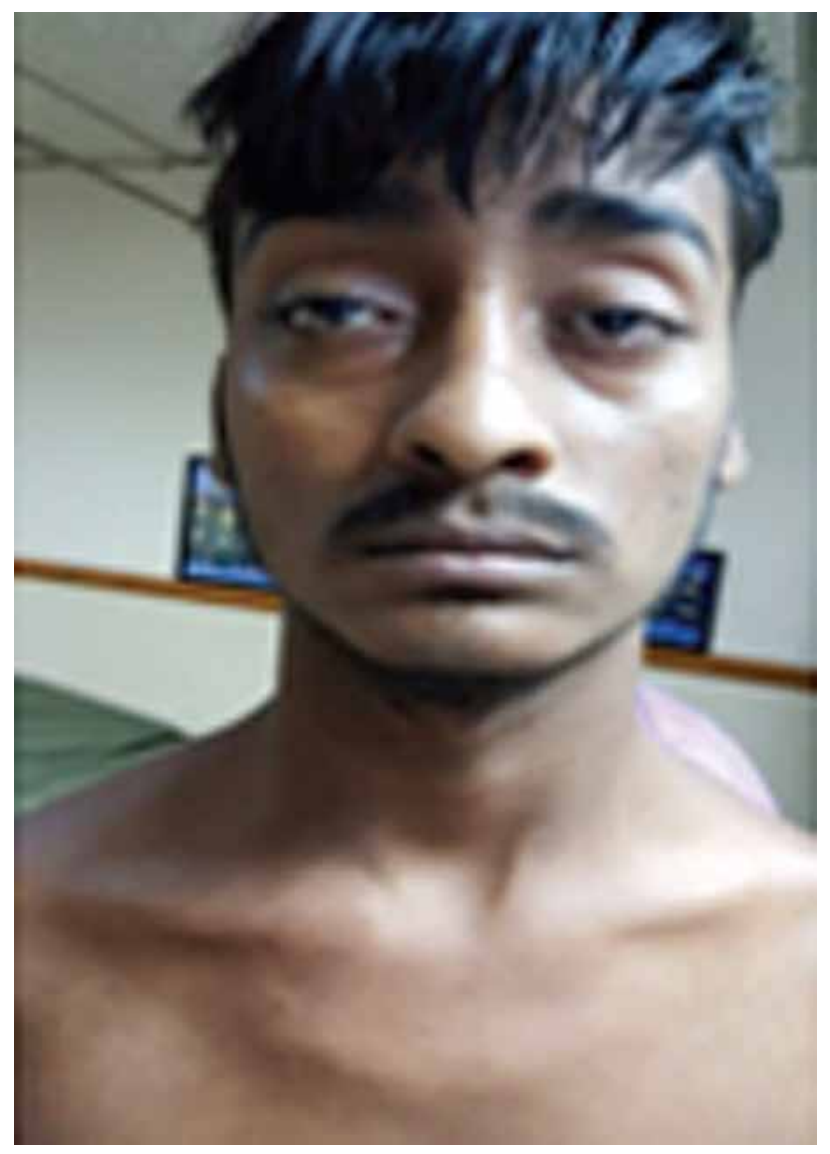

Figure 1: Bilateral ptosis.
Kearns-Sayre Syndrome: A Rare Mitochondrial Disorder

marriage in his family. He is the third issue of his parents. All other family members are enjoying good health.

He has got a long treatment history. He visited several physicians, got hospitalized several times, underwent many noninvasive and invasive, radiological and biochemical investigations, without any specific diagnosis. He was put on several medications previously including vitamin, steroid etc. with no significant improvement.

His general examination was unremarkable with normal vital parameters. Neurological examination revealed intact higher psychic function including speech. There was bilateral partial ptosis (Fig1), complete ophthalmoplegia \& diplopia in all gazes. However, his visual acuity, pupillary light reflex and fundoscopic examination were normal. Motor examination revealed features of lower motor neuron lesion in all 4 limbs as manifested by muscle wasting, hypotonia, reduced muscle power (3/5 both proximally \& distally) and diminished all reflexes. There was winging of scapula (Fig 2).

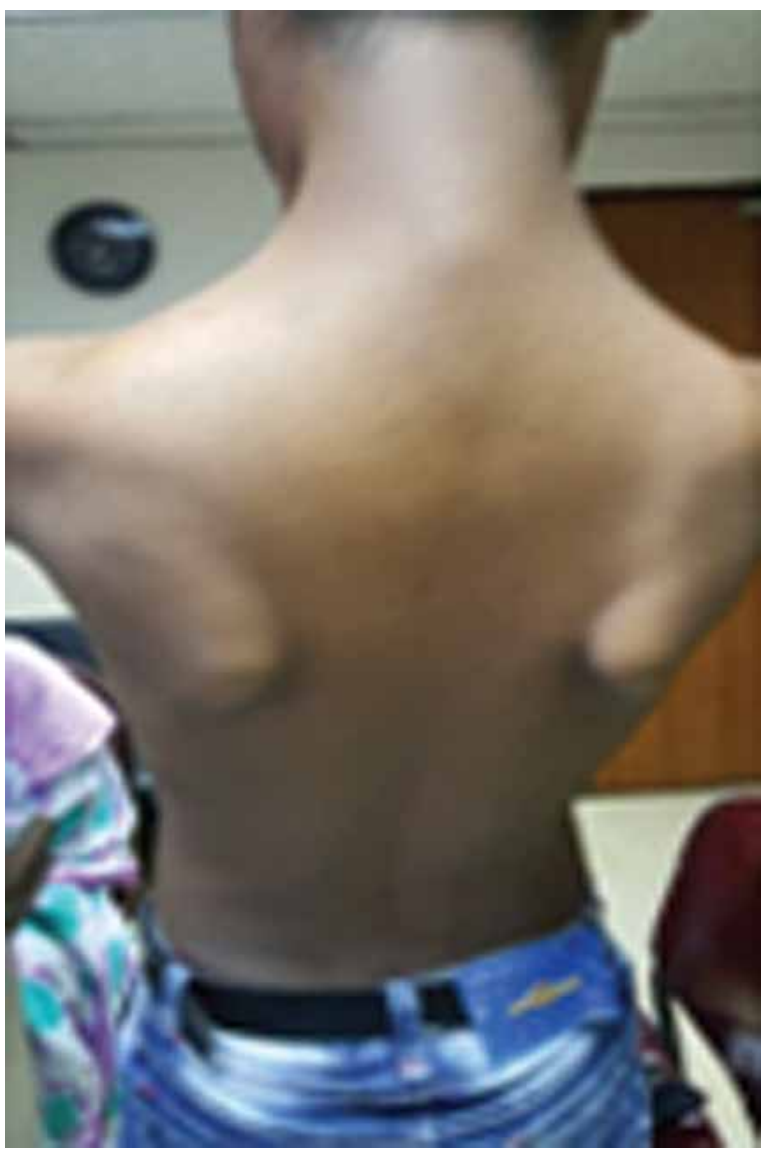

Figure 2: Winging of scapula. 
Regarding cerebellar signs, the patient had trunkal ataxia with ataxic gait. Co-ordination could not be evaluated due to muscular weakness. There was distal sensory impairment bilaterally in all four limbs (in upper limbs - up to elbow, in lower limbs - up to knee joint). Examinations of all other systems were unremarkable.

From history and detailed physical examination, we were confused regarding whether we are dealing with a patient of Myasthenia gravis or myopathy or hereditary neuropathy. To solve the mystery, we did some investigations (Table I). Combination of ptosis, ophthalmoplegia, features of both myopathy and neuropathy with absence of fatigability in a young patient pointed our diagnosis towards mitochondrial disease. Most of the features match with Kearns-Sayre syndrome, although there was no retinitis pigmentosa on fundoscopic examination. Muscle biopsy to detect ragged red fibers and genetic studies to pinpoint the exact nature of the defect in our case could not be done due to lack of facility. As there is no specific treatment available for this condition, conservative management was given with vitamin supplementation, co-enzyme Q10 \& carnitine. Counseling was done regarding the diagnosis and nature of the disease to both the patient and his family members.

\section{Discussion:}

The prevalence of mitochondrial myopathies worldwide was estimated to be $0.025 \%$; however, the accurate frequency of mitochondrial disorders is difficult to estimate because of their clinical and genetic heterogeneity. ${ }^{7}$ Diagnosing mitochondrial disease can be a nightmare, due to the widespread variety and severity of symptoms in the large number of known sub-groups $(>100)$.

Some mitochondrial disorders may affect a single organ only, such as the eye in Leber Hereditary Optic Neuropathy (LHON), but many involve multiple organ systems and often present with prominent neurologic and myopathic features. Many affected individuals display a cluster of clinical features that fall into a discrete clinical syndrome, such as 'mitochondrial encephalopathy with lactic acidosis and stroke- like episodes' (MELAS). However, considerable clinical variability exists and many individuals do not fit nearly into one particular category, ${ }^{8}$ as in our patient. So, the foremost and utmost diagnostic tool is good clinical judgment.

Our patient had features of both myopathy and neuropathy along with ocular involvement sparing pupil. He did not have fatigable weakness like Myasthenia gravis. Combining all the features, we considered him to be a patient of mitochondrial disease, most possibly Kearns-Sayre syndrome. Onset of this Kearns-Sayre syndrome is less than 20 years of age. Symptoms in our patient started at 10

Table I

The important positive findings of laboratory investigation

\begin{tabular}{|c|c|}
\hline Investigations & Findings \\
\hline Creatine Phosphokinase (CPK) & Was done several times, every time there was $2-3$ fold rise from normal value \\
\hline CSF study & CSF protein level was slightly raised with normal cell count \\
\hline $\begin{array}{l}\text { Acetylcholine receptor binding antibody } \\
\text { test (To exclude Myasthenia gravis) }\end{array}$ & Negative $[0.12 \mathrm{nmol} / \mathrm{L}($ Reference value $<0.25)]$ \\
\hline Serumlactic acid & Markedly elevated [150 mg/dl (ref : 3.6-18)] \\
\hline Nerve conduction study ( NCS) & $\begin{array}{l}\text { Suggestive of sensory motor axonal neuropathy. Repeated Nerve Stimulation } \\
\text { of distal single nerve did not show pre/post synaptic defect. }\end{array}$ \\
\hline Skeletal muscle biopsy & $\begin{array}{l}\text { Skeletal muscle reveals foci of perifascicular atrophic muscle fibrous with } \\
\text { inflammatory change compatible with noninfectious inflammatory myopathy. }\end{array}$ \\
\hline CT Scan of brain & Age unrelated cortical atrophy. \\
\hline $\begin{array}{l}\text { Complete blood count, renal function, } \\
\text { thyroid function, blood sugar, serum } \\
\text { electrolytes }\end{array}$ & Within normal limit \\
\hline
\end{tabular}


years of age. Most important features of this syndrome are progressive external ophthalmoplegia (PEO), ptosis, pigmentary degeneration of retina, heart block, myopathy, dysphagia most commonly associated with cricopharyngeal achalasia, hearing loss, ataxia, and dementia. ${ }^{9} \mathrm{He}$ had so many positive features but fundus was normal.

There are no standard guidelines for the investigation of mitochondrial disease. ${ }^{10}$ Investigation is determined by clinical presentation and exclusion of a broad differential diagnosis. No single investigation, except identification of the specific gene mutation, is sensitive or specific to mitochondrial disease. So, for diagnosis, we have to depend on our clinical suspicion and exclusion of other common disorders.

The management of the mitochondrial disease itself is largely supportive ${ }^{11}$, as there is no way of simply increasing the capacity of the cell to generate energy. Treatment therefore involves optimizing energy production, reducing energy losses, meeting lifestyle needs such as education, and monitoring for complications ${ }^{12}$ along with proper counseling of the patient \& his care-givers.

At present there is no known cure and it is not possible to predict the future of a person with mitochondrial disease as the expression of the illness in each individual is extremely variable and difficult to assess. ${ }^{12}$

We can conclude that even though these disorders are chronic and incurable, correct and aggressive early management of many issues can improve the patient's quality of life. For example, at times of stress, early detection or even prevention of a metabolic crisis may limit morbidity and the onset of further handicap.

Conflict of interest: None.

\section{References:}

1. Mitochondrial Disease Information Booklet for
Medical Practitionersby Australian Mitochondrial Disease Foundation. 2014, www.amdf.org.au.

2. Skladal D, Halliday J, Thorburn DR. Minimum birth prevalence of mitochondrial respiratory chain disorders in children. Brain 2003;126:1905-12.

3. Schaefer AM, McFarland R, Blakely EL, et al. Prevalence of mitochondrial DNA disease in adults. Ann Neurol 2008;63:35-9.

4. Milone M, Wong LJ. Diagnosis of mitochondrial myopathies. Mol Genet Metab 2013;110:35-41.

5. Tarnopolsky MA, Raha S. Mitochondrial myopathies: diagnosis, exercise intolerance, and treatment options. Med Sci Sports Exerc 2005;37:2086-2093.

6. Taivassalo T, Haller RG. Exercise and training in mitochondrial myopathies. Med Sci Sports Exerc 2005;37:2094-2101.

7. Wisely NA, Cook PR. General anaesthesia in a man with mitochondrial myopathy undergoing eye surgery. Eur J Anaesthesiol 2001;18:333-335.

8. Chinnery P.F. (2006). Mitochondrial disorders overview GeneReviews

9. Naviaux, R.K. A Primary Care Physician's Guide, the Spectrum of Mitochondrial Disease. United Mitochondrial Disease Foundation at www.umdf.org

10. Haas RH, Parikh S, Falk MJ, et al. The in depth evaluation of suspected mitochondrial disease. Mol Genet Metab 2008;94:16-37.

11. Chinnery P.F. \& Turnbull D.M. Epidemiology and treatment of mitochondrial disorders. Am j Med Genet. 2001;106:94- 101 .

12. A Clinician's Guide to the management of Mitochondrial Disease: A manual for primary care providers developed by Margaret Klehm, Mark Korson, and mitoaction.org, 2008, www.mitoaction.org. 